\title{
Un asunto superficial: el revoco decorativo del recinto amurallado romano-medieval de León
}

\author{
Julio M. Vidal Encinas \\ María Luz González Fernández \\ Rocío Fernández Ordás \\ José C. Álvarez Ordás \\ Pablo Rodríguez González
}

RESUMEN. Se describe un revoco decorativo a base de fajas horizontales rellenas de líneas oblicuas en el recinto amurallado de León, así como en el muro que rodea el antiguo castellum. Los autores enfatizan sobre la importancia que tiene, como documento histórico y patrimonial, tal elemento decorativo, cuyo origen debe asociarse a la construcción de la Cerca (fines del s. XIII-XIV). En algunos casos, ciertas evidencias proporcionadas por estos revocos parecen sugerir la anterioridad de algunos elementos del recinto - caso de una de las torres de Puerta Moneda - con la implantación de la misma Cerca.

Palabras clave: Muralla, Medieval, revoco.

ABSTRACT. This paper report the existence of a decorative plastering in the Romano-Medieval Wall of León, as well as in the wall that surrounds the old castellum. The authors emphasize the importance of such decorative element as historical document, whose chronology seems that it must be associated to the uprising of the Wall (end of s. XIIIXIV), and, in some cases, even previous to the construction of certain sections, at least in the zone of Puerta Moneda.

Key words: Wall, Medieval, plastering.

“Las murallas son (...) el símbolo de todo lo que puede haber de corroboración y exaltación, pero también de paralización, en el patrimonio monumental de una ciudad" (J. LE GOFF)

\section{INTRODUCCIÓN}

Una de las cuestiones más desconocidas para el público en general -y, en buena parte, también para los investigadores-, es el aspecto exterior que los monumentos antiguos tuvieron para sus contemporáneos.

En efecto, como consecuencia del paso del tiempo y de modas "restauradoras" muy recientes, la mayor parte de las obras más señeras de nuestro Patrimonio Histórico nos presentan sus muros o paramentos externos limpios de los revocos y revestimientos que, en origen, poseyeron, cuando, en realidad, los constructores clásicos o medievales, por ejemplo, nunca los concibieron para mostrar su "fábrica vista", "descarnada", como hoy la vemos. 
Tal estampa es tan alejada de la realidad original que, si en la actualidad se restaurara, el resultado sería tan chocante que difícilmente podría ser aceptado por los ciudadanos, sean cuales fueran sus conocimientos culturales. ¿Quién podría hoy aceptar que al Partenón se le devolviera la piel que lo cubría por completo, pintada con colores sumamente vistosos -azules, dorados, amarillos, rojos...-, que hoy nos parecerían aparatosos y estridentes, próximos al kitsch? ¿Puede alguien imaginarse una catedral gótica recubierta de purpurina dorada? Acostumbrados, como estamos, desde el redescubrimiento que el romanticismo hizo del Arte Antiguo, a la observación directa de la "noble" piedra, ésta constituye, por antonomasia, el elemento material que nos ayuda a identificar la enjundia histórica de cualquier construcción que se precie. Algo a lo que ha contribuido un cierto concepto de restauración, paradójicamente "historicista" -pero, a la postre, ignorante de la antigua realidad histórica de los monumentos que pretendía restituir-, que se dedicó a arrancar sus revocos y enlucidos interiores y exteriores, provocando, en el primero de los casos, la pérdida de importantes motivos decorativos pictóricos en ellos ${ }^{1}$. Pero las consecuencias de tan inadecuadas intervenciones no deben solamente medirse en términos de pérdidas, digamos, "artísticas", aún cuando a nadie se le escapa que muchos de los atolondrados picados que se han efectuado en el interior de ciertas iglesias medievales, por ejemplo, hayan podido llevarse consigo relevantes motivos pictóricos. Deben medirse, también, en términos de desaparición

\footnotetext{
${ }^{1}$ Sobre la importancia que, desde el punto de vista arqueológico y arquitectónico, tienen los revestimientos en los edificios históricos ver: I. ARCE, "El estudio de los acabados y revestimientos de la arquitectura", Actas del Simposio: Arqueología de la Arquitectura. El método arqueológico aplicado al proceso de estudio y de intervención en edificios históricos, Burgos, 1996. Junta de Castilla y León, 87-102.
}

de información histórica muy valiosa para conocer las vicisitudes por las que han pasado los edificios: la superposición de diferentes revocos pintados, por ejemplo, nos pueden informar de las etapas constructivas que han tenido, así como de los conceptos decorativos que las han regido. El revoco, además, ha sido soporte tradicional para algo tan aparentemente nimio como los grafitos, cuya información, las más de las veces, sobrepasa el ámbito estrictamente circunstancial o pintoresco. Pensemos, sin ir muy lejos, en el valor que deben tener están sin estudiar, todavía..., a pesar de haber sido descubiertos hace más de diez años-, los numerosos grafitos medievales que existen en la epístola y evangelio de la iglesia mozárabe de Santiago de Peñalba². Elementos, todos ellos, aparentemente "menores", pero que no cabe duda que enriquecen el monumento.

Pero es que, además, esta cualidad -la existencia de "piel" en los edificios antiguos-, no sólo se constataba en aquellos levantados en piedra -fuera cual fuera su forma de aparejarla-, sino que debe extenderse también a aquellos levantados en ladrillo. Contrariamente a lo que habitualmente se cree, la Arquitectura Romana, por ejemplo -si exceptuamos ciertos edificios públicos-, empleó de una forma mucho más habitual el ladrillo, el cual, precisamente, se enfoscaba y pintaba. Con ello se conseguía, por un lado, proteger los paramentos de los agentes externos $\mathrm{y}$, por otro, realzarlos por medio del color y, también, de la decoración.

\footnotetext{
2 Una noticia sobre estos grabados en: J.M. VIDAL ENCINAS, "Los grabados murales de la iglesia mozárabe de Santiago de Peñalba". Ier. Congrés Internacional de gravats rupestres $i$ murals. Lleida, 1992 (Resums de Ponències i comunicacions), p.30. Este edificio tiene otra relevante particularidad, cual es la de conservar parte de sus pavimentos originales de opus signinum, una argamasa de origen romano.
} 
Pues bien, construcciones tan sumamente monumentales como son los recintos amurallados de nuestras actuales ciudades históricas no fueron ajenos a tales prácticas. Sin ir más lejos, dos de las murallas romanas de León muestran las huellas de haber sufrido tratamientos en su superficie. La más antigua, y menos conocida, presenta juntas realzadas por medio de un encintado de mortero entre los sillares, que, quizás, se pintaron ${ }^{3}$, como también sucede en uno de los monumentos más señeros de la Tardía Antigüedad en León, el Mausoleo o Basílica Paleocristiana de Marialba de la Ribera, en Villaturiel ${ }^{4}$. La muralla más reciente, por su parte, bien conocida por sus altas torres y lienzos, debió estar enteramente revocada, como así parece apuntarlo el enlucido de argamasa que, en los paños que no han sido objeto de restauración alguna, desborda las juntas entre los mampuestos, algo que, probablemente, está indicando que debía cubrirlos por completo. Sobre tal revoco acaso se aplicó una capa pictórica en la que, por analogía con otros recintos campamentales romanos, se pudo haber perfilado un despiece de sillería regular, imitando una fábrica de tales características. Así se muestra, por ejemplo, en la moderna restauración -más bien reconstrucción- del campamento romano de Saalburg, en los Montes Taunus, en Alemania, en la que se ha cuidado de reproducir tal revestimiento en una pequeña parte de su muralla.

${ }^{3}$ Un tramo actualmente visible de la misma se encuentra a la izquierda de la escalinata que sube desde la calle Ramón y Cajal a la Plaza de San Isidoro. Ver: A. GARCía y BELLIDO, Nueve estudios sobre la Legio VII Gemina y su campamento en León, 1968, León, 11-16.

${ }^{4}$ T. HAUSCHILD, "La iglesia martirial de Marialba (León)”, Tierras de León, 9, 1968, 21-26. (p. 23)

\section{CARACTERÍSTICAS CONSTRUC- TIVAS E HISTORIA DE LA CERCA MEDIEVAL DE LEÓN}

La Cerca Medieval de León, por su parte, así como la muralla romana -en las reparaciones o reconstrucciones de que ha sido objeto, a base de fábricas de cal y canto- no fueron ajenas a tales particularidades -la existencia de un tratamiento de revoco decorativo en su superficie-, en las que, en nuestro conocimiento, no se había reparado debidamente, especialmente en sus implicaciones históricas o cronológicas, que, como veremos, son sustanciales ${ }^{5}$.

No existe todavía, por sorprendente que pueda parecer, un estudio detallado sobre los recintos amurallados romanomedievales de León, tanto en sus aspectos estrictamente arquitectónicos y arqueológicos, como documentales, especialmente en lo que se refiere al más moderno de ellos6. En consecuencia, siguen constituyendo fuente imprescindible publicaciones que, en algunos casos, se remontan a treinta años

\footnotetext{
${ }^{5}$ Existe una alusión a estos enfoscados en: C. SEXMILO HUARTE, Documentación técnica para la restauración de las murallas de León en el tramo calle Carreras - Av. de los Cubos, 1993 ( Informe inédito depositado en el Servicio Territorial de Cultura de León, p.15), cuyo estudio de estratigrafía muraria fue realizado por el arqueólogo E. Campomanes. Sin embargo, dicho estudio asigna una cronología "del siglo XIX" a todas las fábricas de canto rodado en la que tales enlucidos se encuentran (p. 5) y no establece ninguna recomendación expresa sobre el interés de su conservación.

${ }^{6}$ Sobre los aspectos históricos del tema ver: J. VALDEÓN BARUQUE, "Reflexiones sobre las murallas urbanas de la Castilla Medieval", La Ciudad y las Murallas, C. De SeTA Y J. Le Goff (eds.), 1991, Madrid, 67-87. A reseñar también un interesante trabajo sobre este tema, recientemente aparecido: F. BENITO MARTíN, La formación de la ciudad medieval. La red urbana en Castilla y León, 2000, Universidad de Valladolid, 148-150 y passim.
} 
atrás ${ }^{7}$ o bien otras que, más recientes, revisan y compendian aportaciones anteriores ${ }^{8}$.

La Cerca Medieval de León, o "del Burgo Nuevo", se desarrolló hacia el sur del recinto campamental romano, englobando todo su lienzo meridional y un tercio del occidental. Sus aproximadamente 15 Ha. de superficie se cerraban, a occidente, un poco al norte de su porta principalis dextra, conocida como Cauriense y, a oriente, en la Turris Quadrata o Torreón de los Ponce, situada en la esquina sudoriental del antiguo castra de la Legio VII Gémina. Su edilicia se resume en un muro de unos 3 metros de anchura, levantado por medio de un encofrado de cal y canto, provisto de adarve y pretil almenado; una liza de, según los lugares, entre 3,5 y 4 metros de anchura y, finalmente, una barrera de 1,75-2 metros de anchura, también provista de adarve, parapeto y almenas. Cerca y barreras alcanzan, respectivamente, 8 y 4 metros de altura y, éstas últimas, no parecen estar presentes en todo su trazado', que alcanza, para toda la fortificación medieval, unos $1.300 \mathrm{~m}$. de longitud. Tuvo, a lo largo de su historia, 9 puertas $y$ postigos con diferentes denominaciones ${ }^{10}$, de las que apenas resta

${ }^{7}$ A. REPRESA, "Evolución urbana de León en los siglos XI-XIII", León y su Historia, t. I, 1969, León, 243282; I. GONZÁLEZ GALLEGO, "Las murallas y los puentes de León en el siglo XIV". León y su Historia, t. IV, 1977, León, 365-411; , E. BENITO RUANO, “Las Murallas y Cercas de la Ciudad de León durante la Edad Media". León Medieval. Doce Estudios, 1978, 25-40.

${ }^{8}$ C. Álvarez Álvarez, La ciudad de León en la Baja Edad Media. El espacio urbano, 1992, León; J. A. GUTIÉRREZ GONZÁLEZ, Fortificaciones y Feudalismo en el origen y formación del Reino Leonés (siglos IX-XIII), 1995, Universidad de Valladolid, 235-249.

${ }^{9}$ Así parece sugerirlo el resultado de unos sondeos realizados en 2001 por el Arqueólogo Municipal de León, Victorino García Marcos, en una zona del paño oriental situada entre la Puerta del Sol y el Torreón de los Ponce, puesto que en la trinchera abierta por la máquina excavadora no se registró resto de construcción alguna. naciones ${ }^{10}$, de las que apenas resta nada, salvo una de las torres de Puerta Moneda en lamentable estado de conservación- y, quizás, de otra de las torres de la Puerta de Santa Ana o Cal de Moros, embutida en una vivienda actual ${ }^{11}$.

Desde un punto de vista cronológico, la Cerca Medieval, en su formalización actual -salvo en lo que atañe a las diferentes obras de adecuación posteriores de que fue objeto su coronación o almenado y aún restauraciones ${ }^{12}$, , parece ser obra de finales del siglo XIII o de la primera mitad del siglo $\mathrm{XIV}^{13}$, si bien su origen se remonta dos siglos más atrás, puesto que desde finales del siglo XII existen ya referencias a la existencia de un murus terrae, construido en tapial. En efecto, éste habría sido alzado para encerrar la aglomeración urbana sur-

10 Puerta de Cal de Escuderos, del Peso o Torre del Obispo; de Diego Gutiérrez o del Caño Badillo; del Sol; de Cal de Moros o Santa Ana; Moneda; Gallega o de San Francisco; del Burgo Nuevo o de las Ánimas; de Santo Domingo o Fajeros y Postigo de la Ollería.

${ }^{11}$ A pesar de la pintura que presenta la vivienda que hace esquina en la Cuesta de Castañones con Plaza de Riaño se puede observar un pequeño fragmento de revoco decorativo en la fachada que coincide con el trazado de la Cerca.

${ }^{12}$ La última tiene lugar en el siglo XIX, con motivo de las Guerras Carlistas. Sin embargo, J.A. GUTIÉRREZ GONZÁLEZ, op.cit. 239, atribuye a la Baja Edad Media estas reformas en la coronación de la Cerca, consistentes, según su descripción en "... saeteras (...) enmarcadas en ladrillo, con viguetas de madera (...)", cuando, en realidad, son aspilleras para fusilería que se abren entre 1836 y 1841. Ver, al respecto: Expediente General de Fortificación y Libros de Actas y Acuerdos Municipales de 1836-1841, depositados en el Archivo Histórico Municipal de León. También: M.L. GonZÁLEZ y R. FERNÁNDEZ ORDÁs, Lectura muraria de la Cerca Medieval de la ciudad de León, 2001 (Informe inédito. Junta de Castilla y León. Servicio Territorial de Educación y Cultura). La denominada calle de Las Cercas, entre Puerta Moneda y Puerta de Santa Ana, fue restaurada por el arquitecto Luis Menéndez Pidal en 1968 y el paño de la calle Independencia, una vez liberado de las viviendas adosadas, se restauró en 1985-1986.

${ }^{13}$ Represa, op. cit., passim; González Gallego, 393- 
gida al socaire del Camino de Santiago -el burgo de francos de la rua o vico francorum-, $\mathrm{y}$ en torno a las actividades del mercado del Barrio de San Martín, de los que se tiene noticias desde, al menos, el siglo $\mathrm{XI}^{14}$. Así, por ejemplo, existen documentos que aluden a alguna de las puertas de tal recinto Puerta Moneda y Puerta Gallega-, que se remontan a 119915 .

\section{EL REVOCO DECORATIVO DE LA CERCA MEDIEVAL DE LEÓN (Fig. 1)}

Con motivo de recientes (2001) trabajos de mejora y restauración de la liza existente en el paño más meridional de la Cer$\mathrm{Ca}^{16}$, entre las Puertas de Santa Ana y Puerta Gallega, hemos tenido ocasión de realizar ciertas observaciones a la altura de la puerta intermedia, Moneda, que están en el origen de estas breves notas. Esta puerta, como más arriba se señalaba, es mencionada en la documentación histórica desde finales del siglo XII y debe su nombre a la instalación de los monederos o fabricantes de

14 RePresA, op. cit., 255; GONZÁlez GALleGo, op. cit., 375 y ss.; también: C. ESTEPA, Estructura social de la ciudad de León, Fuentes y Estudios de Historia Leonesa 19, 1977, León, 129 nota 109, 142 y 143.

15 REPRESA, op. cit., 259.

${ }^{16}$ Los trabajos fueron promovidos por el Ayuntamiento de León. Paralelamente a ello se llevó a cabo un estudio del paramento de la zona de muralla a intervenir y de supervisión arqueológica de las obras, bajo la dirección de dos de los firmantes, María Luz González Fernández y Rocío Fernández Ordás, con aportaciones de los arquitectos Ramón Cañas Aparicio y Carlos Sexmilo. Los resultados de estos trabajos se encuentran en los informes: Lectura muraria de la Cerca Medieval de la ciudad de León y Seguimiento y documentación arqueológica en el proyecto de urbanización de la calle de Las Cercas entre la plaza de San Francisco y la plaza de Riaño, en León. 2001. (Informes inéditos depositados en el Servicio Territorial de Cultura de la Junta de Castilla y León). Queremos desde aquí expresar nuestro agradecimiento a la empresa adjudicataria de las obras, Decolesa, y, en particular, a D. Carlos Fernández, por la ayuda y comprensión prestadas. moneda en sus inmediaciones, concretamente en el primer tramo urbano de la rua francorum, al igual que los cambiadores, también situados en su proximidad ${ }^{17}$.

La decoración que registramos consiste en el trazado de unas bandas horizontales, paralelas, de unos 20-30 cms. de anchura, practicadas en el revoco aún fresco que cubre la mampostería de canto rodado, las cuales se rellenaron, también utilizando la punta de la paleta o llana, con incisiones oblicuas que ocupan toda la faja. En algunos casos existe una cierta regularidad pero, en otros, no hubo mucho interés en buscarla. Los lugares en los que hasta el momento han podido observarse tales revocos decorativos son ${ }^{18}$ :

\section{a) Zona de Puerta Moneda}

Se observa en dos zonas diferentes. En primer lugar, en el costado oriental de la misma torre (Fig. 2), en la que ocupa la fábrica de canto rodado inmediata a la esquina de sillería, en la que hemos podido registrar dos marcas de cantero con el signo $\llcorner$. Está parcialmente oculta por el paño de la muralla que se entrega a la misma, dejando una separación bien neta, lo que quiere decir que ambas fábricas -torre $\mathrm{y}$ cerca-, no traban entre sí19.

En segundo lugar, se encuentra este enfoscado decorado en el tramo bajo de la misma Cerca, en la cara que acometía a la

\footnotetext{
${ }^{17}$ ESTEPA, op. cit., 398 y 399.

${ }^{18}$ Existe otro lugar en el que, muy recientemente, se han podido constatar estos revocos y sobre el que no nos extenderemos por ser en la actualidad objeto de excavaciones arqueológicas, aún no finalizadas. Se trata de un solar sito en la Cuesta de Castañones, en el que se han hallado restos de una construcción enlucida con estas decoraciones. Los trabajos se realizan bajo la dirección de Pablo Rodríguez González.

${ }^{19}$ Este hecho, no obstante, a veces se interpreta como intencional por los constructores, para impedir que la caída de una torre arrastre consigo al lienzo.
} 
Torre, lo que no deja de ser curioso, ya que se trata del negativo o impronta que quedó impreso en las rebabas de argamasa que se originaron al utilizar esta cara de la torre como límite del encofrado que sirvió para levantar la Cerca (Fig. 3). Por lo tanto, en origen, tal revestimiento decorado pertenecía a la Torre -concretamente al macizo en el que se construyó la escalera para subir al adarve de la muralla-, lo que quiere decir, por un lado, que tal torre ya estaba en pie cuando se levantó aquella, y, por otro, que, como tal decoración, se concibió para ser vista, por lo que el paño de muro al que se asociaba no debía tener la implantación que tiene el actual, ya que no permitiría su contemplación. La zona en la que se puede observar este detalle corresponde a la parte baja de la torre, allí dónde se sitúa la escalera de subida al adarve de la Cerca, la cual conserva todavía los dos primeros peldaños de su comienzo y final. Tal escalera fue demolida -al igual que la parte superior de la muralla-, por causa de una vivienda que se adosó a la misma, lo que ha permitido exponer tal revoco. Las condiciones en las que se ha observado este revoco de Puerta Moneda permite, en nuestra opinión, asociarlo de forma fehaciente a la construcción de la Cerca.

\section{b) Muralla correspondiente al recinto romano}

Sus lienzos y torres presentan en la actualidad una gran heterogeneidad pero, en sentido amplio, se suele comúnmente identificar con la fábrica original romana aquella que muestra paramentos de opus incertum, formados por bloques de cuarcita, caliza y arenisca, de variados tamaños, frente a aquellas superficies constituidas mayoritariamente por canto rodado, atribuidas a época medieval. La documentación histórica viene a avalar restauraciones medievales realizadas en cal y canto en la muralla romana desde comienzos del siglo
XIII y, concretamente, los recrecidos que se pueden observar a base de tales materiales en la Avda. de los Cubos se atribuyen a este momento por algún autor ${ }^{20}$.

Los enlucidos decorados los encontramos, en primer lugar, en el tramo que discurre por la calle Carreras y Avda. de los Cubos, sobre todo en varias de las torres que se encuentran en esta última, en las que se asocian, indefectiblemente, a fábricas de cal y canto, visibles en sus tramos superiores. En segundo lugar, se registran en la calle Serradores, en dos de los cubos que se encuentran en el tramo que va desde Puerta del Obispo hasta la Torre de los Ponce o Quadrata, como es denominada en la documentación medieval, al menos desde el siglo $\mathrm{XII}^{21}$. Pequeños restos se pueden también contemplar en una de las torres inmediata a la contemporánea Puerta de Regla y en un pequeño paño de muralla situado entre ésta y el edificio conocido como el “Torreón" 22.

El primitivo recinto de Legio parece que fue restaurado en el siglo XI, si bien existe cierta confusión acerca del monarca que acometió tales obras, Alfonso $\mathrm{V}$ o Fernando $\mathrm{I}^{23} \mathrm{y}$, no menos, sobre los materiales empleados en las puertas, "ex luto et ligneo", a saber, tapial o adobe y madera, al tratarse de una imponente muralla de mampostería trabada con argamasa, como todas las romanas. Sea como fuere, lo cierto es que, en los recrecidos medievales de las primeras torres romanas, inmediatas a la esquinas nororiental y sudoriental del recinto, se puede apreciar claramente la existencia de

-

${ }^{20}$ Por ejemplo, GutiérReZ GONZÁLEZ, op. cit., 245, que, sin embargo, no repara en la existencia de los revocos.

${ }^{21}$ ESTEPA, op. cit., 125.

22 Este edificio muestra numerosas y variadas marcas de cantero, entre ellas una idéntica $(L)$ a la de Puerta Moneda, a la que aludimos más arriba.

${ }^{23}$ REPRESA, op. cit., 251, nota 19. 
estos enfoscados decorativos, que cubren la fábrica de cal y canto, ello a pesar de recientes intervenciones públicas, supuestamente "restauradoras", pero, a la postre, lisa y llanamente, destructoras ${ }^{24}$.

c) Cerca que rodea el antiguo castellum, castrum o turres, del comes primero, luego tenente, de la ciudad (actual Archivo Histórico, inmediato a Puerta del Conde o Puerta Castillo)

Desde el siglo X se conoce la existencia en León de un tal delegado del poder regio, el cual tendría su residencia en las inmediaciones de la Porta Decumana o septentrional del antiguo recinto romano, para lo cual, probablemente, se habilitarían sus torres, primero, y, más tarde, los dos cubos inmediatos en este paño de la muralla. Éstos -más una torre de sillería plenomedieval (¿?) que engloba, probablemente, otra anterior-, se presentan actualmente cercados por un alto muro de cal y canto de factura similar a cualquiera de las fábricas de la Cerca Medieval, o a los recrecidos de la misma época realizados sobre las torres romanas que presentan revocos decorativos. Sin embargo, la cerca que rodea el antiguo castellum es considerada por algún autor como un revellín'25, añadido, incluso, en el siglo $\mathrm{XVI}^{26}$, filiación funcional y cronológica que, quizás, habría que reconsiderar a la luz de los datos que aquí aportamos.

-

${ }^{24}$ Nos estamos refiriendo a obras llevadas a cabo por el Ayuntamiento de León en octubre de 2000 en la Avda. de los Cubos, que acarrearon la desaparición de buena parte de estos enfoscados en, al menos, una de las torres.

${ }^{25}$ M. GÓMEZ-MORENO, 1925: Catálogo Monumental de la Provincia de León. Ministerio de Instrucción Pública (Ed. Facsímil, Editorial Nebrija, 1979, León), p. 176.

${ }^{26}$ J.A. GUTIÉRREZ, op. cit., 239, repite tal adscripción. En realidad un revellín - hoy desaparecido -, se encontraba, como todas las obras de esta índole, por delante de la fortificación y aislado. Así se puede comprobar en el Plano del Recinto Magistral de León, de 1835.

\section{PRESENCIA DE REVOCOS DECO- RATIVOS EN OTROS EDIFICIOS HISTÓRICOS DE LA CIUDAD DE LEÓN}

Recientemente hemos tenido ocasión de constatar la existencia de estas decoraciones en un edificio muy singular del Conjunto Histórico de León, el denominado "Palacio o Torre de Doña. Berenguela", una construcción de índole civil que se encuentra en un estado de total desvalorización a causa de su extravagante situación: el patio de recreo de un colegio de enseñanza, el de las MM. Teresianas, próximo a la Catedral. Aquí, gracias a un documento fotográfico de fecha imprecisa ${ }^{27}$, pero que debió tomarse en la primera mitad del siglo $X X$, se pueden apreciar perfectamente estas decoraciones (Figs. 4 y 5). El enfoscado que les sirvió de soporte, sin embargo, no existe en la actualidad, eliminado por alguna de las últimas restauraciones que sufrió el edificio. Sea como fuere, lo cierto es que sus características arquitectónicas parecen corresponder a las de un románico avanzado, quizás de finales del siglo XII o comienzos del XIII, de la época del reinado de Alfonso IX $(1188-1230)^{28}$.

\section{v. CONCLUSIONES}

1a. El recinto amurallado de León conserva un precioso documento histórico representado por estos revocos decorativos, los cuales es preciso investigar adecuadamente, $\mathrm{y}$, sobre todo, mantener y proteger, como muestra inequívoca del revestimiento original que poseyó su antigua fortificación.

${ }^{27}$ Agradecemos a D. Wenceslao Álvarez Oblanca, responsable de la "Institución Fray Bernardino de Sahagún", de la Diputación Provincial de León, las facilidades para reproducir este documento.

${ }^{28}$ C. COSMEN ALONSO, E. FERNÁNDEZ GONZÁLEZ Y M. VALDÉS FERNÁNDEZ, 1990: “La arquitectura románica". Historia del Arte en León. Editado por Diario de León, 70-71. 
Su valor viene también acrecentado por su singularidad, ya que no son muy numerosos los recintos amurallados en España que aún los conserven. En León aparecen siempre asociados a fábricas de cal y canto.

$2^{\mathrm{a}}$. De acuerdo con las observaciones realizadas en los existentes en Puerta Moneda, todo parece indicar que la torre conservada es anterior al muro de la Cerca, cuyo trazado no parece corresponder al de la implantación de dicha torre. Ello parece plantear interesantes interrogantes sobre la cronología de la misma -por ejemplo, que perteneciera al primitivo recinto del burgo nuevo, documentado desde el siglo XII, o a otro momento de un proceso constructivo muy dilatado-.

En cualquier caso, sólo una investigación detallada del recinto medieval de León, cada vez más necesaria, podrá resolver estos interrogantes.

$3^{3}$. La presencia de estos revestimientos en la cerca que rodea el antiguo castellum, en Puerta del Conde o Castillo, anima a proponer la hipótesis de que tal amurallamiento pudiera ser más antiguo medieval- que la cronología que se ha propuesto, de comienzos de la Edad Moderna. 

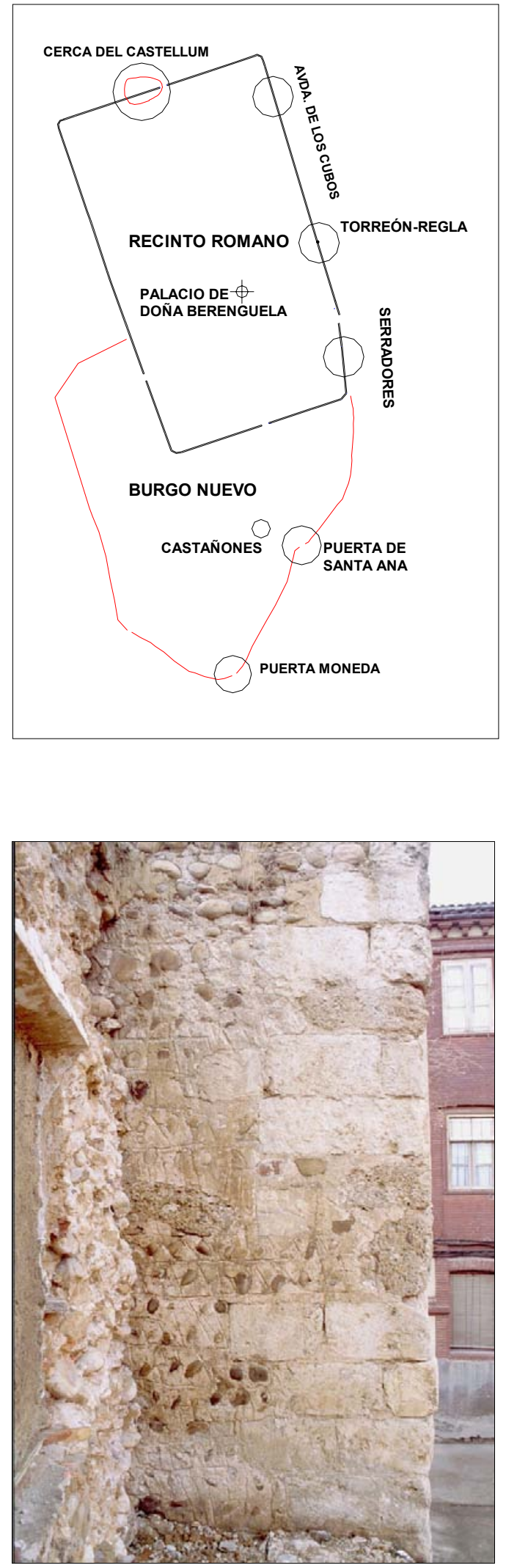

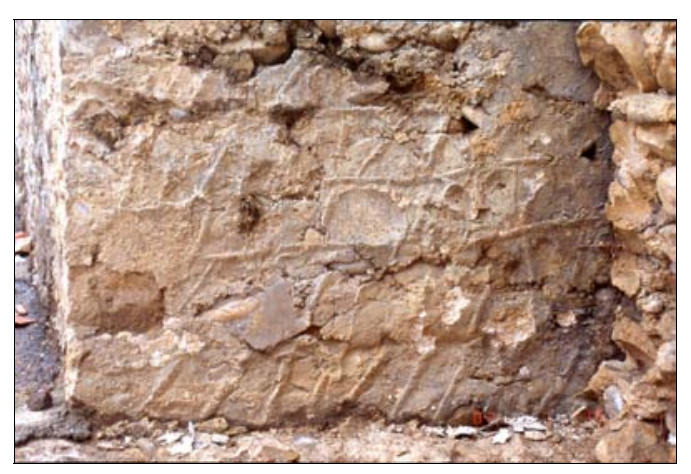

- Fig.1. León. Recintos amurallados romano y medieval. Situación de alguno de los lugares en los que se han registrado revocos decorativos. (arriba izquierda)

- Fig. 2. Puerta Moneda. Torre oriental. Revocos decorativos visibles en la parte inferior y central de la imagen, en la fábrica de canto rodado, a causa de la demolición de la Cerca para instalar una vivienda sobre ella.

(abajo izquierda)

- Fig. 3. Puerta Moneda. Impresión en negativo de los revocos decorativos en la Cerca medieval, al ser utilizada la torre de la Puerta como encofrado. La demolición de un tramo de la escalera de acceso al adarve ha expuesto tales revocos.

(arriba derecha) 


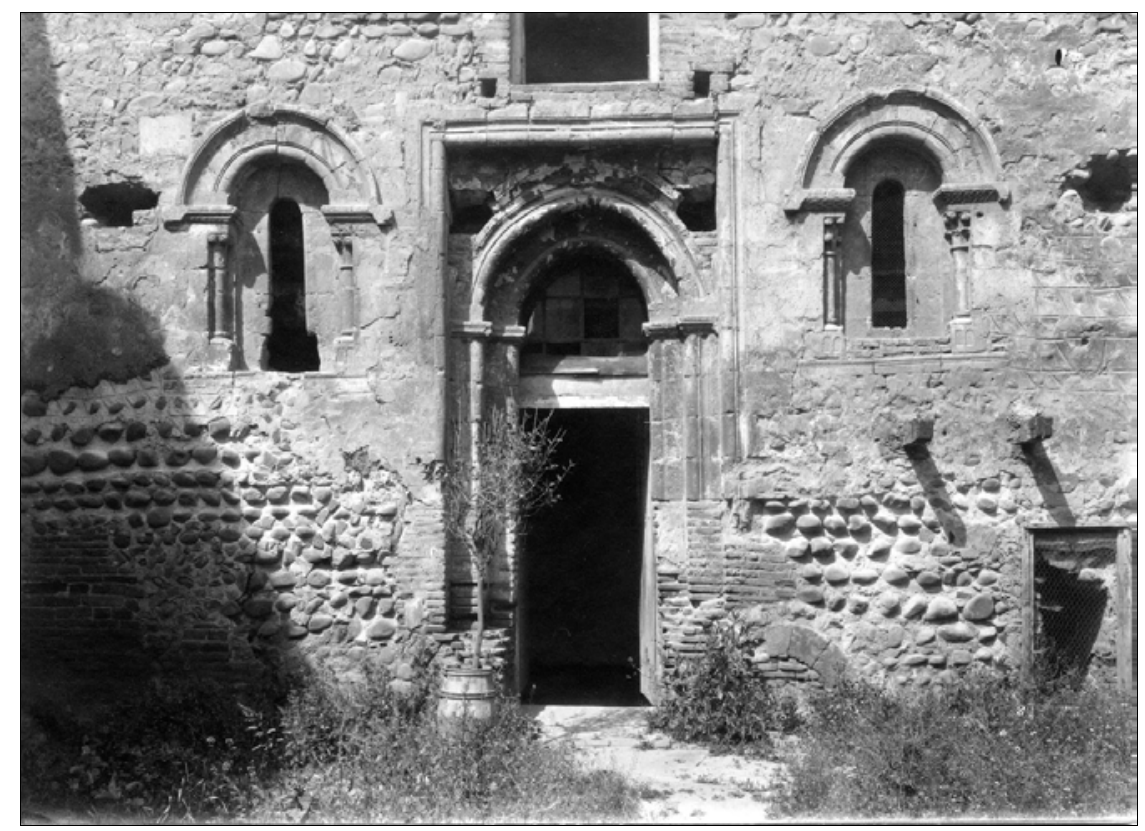

- Fig. 4. Palacio o Torre de Doña Berenguela. Los revocos decorativos se encuentran en el borde derecho de la imagen, a la altura de la parte media e inferior de la ventana.

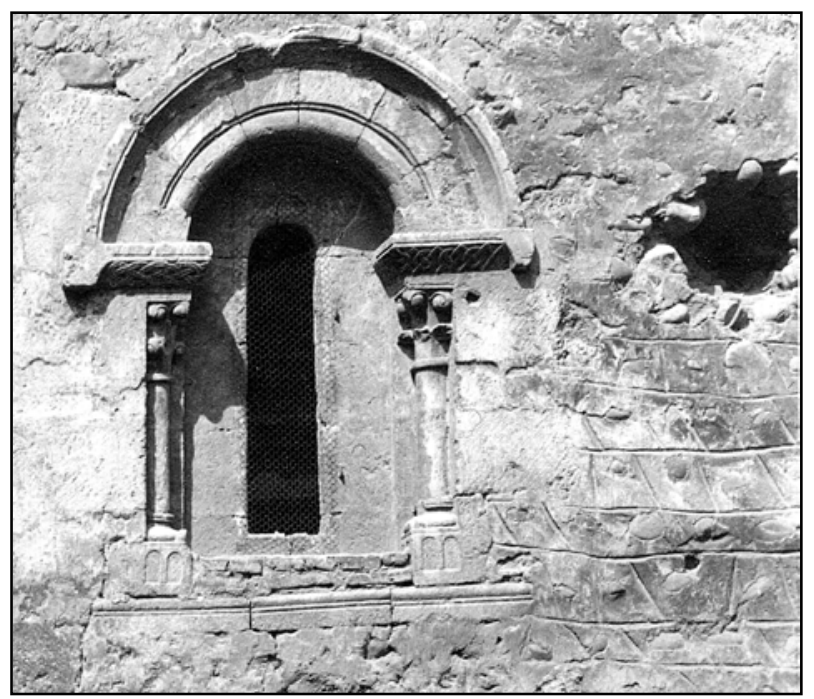

- Fig. 5. Detalle de la imagen anterior. 